\title{
Histopathologically Proven Autoimmune Pancreatitis Mimicking Neuroendocrine Tumor or Pancreatic Cancer
}

\author{
Shinji Onda ${ }^{a}$ Tomoyoshi Okamoto $^{a}$ Masaru Kanehira $^{a}$ \\ Shuichi Fujioka ${ }^{a}$ Tohru Harada $^{c}$ Hiroshi Hano $^{c}$ \\ Masaharu Fukunagab Katsuhiko Yanaga ${ }^{d}$ \\ Departments of a Surgery and ${ }^{b}$ Pathology, The Jikei University, Daisan Hospital, \\ and Departments of ${ }^{\mathrm{C}}$ Pathology and ${ }^{\mathrm{d}}$ Surgery, The Jikei University School of \\ Medicine, Tokyo, Japan
}

\section{Key Words}

Differential diagnosis · Endoscopic ultrasonography · Fine-needle biopsy · IgG4

\begin{abstract}
Autoimmune pancreatitis (AIP) can be difficult to distinguish from pancreatic cancer. We report a case of histopathologically proven AIP mimicking neuroendocrine tumor (NET) or pancreatic cancer in a 53-year-old man. He was referred to our hospital for further evaluation of a pancreatic mass detected on ultrasonography at a medical check-up. Abdominal ultrasonography showed a 15-mm hypoechoic mass located in the pancreatic body. Computed tomography revealed a tumor without any contrast enhancement, and magnetic resonance imaging demonstrated the mass to be hyperintense on diffusion-weighted image. Endoscopic retrograde cholangiopancreatography revealed slight dilatation of a branch of the pancreatic duct without stricture of the main pancreatic duct. The common bile duct seemed intact. Under suspicion of a non-functioning NET or malignant neoplasm, laparotomy was performed. At laparotomy, an elastic firm and well-circumscribed mass was found suggestive of a non-functioning NET, thus enucleation was performed. Histopathologically, the lesion corresponded to AIP.
\end{abstract}

\section{Introduction}

Autoimmune pancreatitis (AIP) is a type of chronic pancreatitis characterized by an autoimmune inflammatory process in which prominent lymphocyte infiltration with associated fibrosis of the pancreas causes organ dysfunction. AIP is a rare disease. 
Although there has been an increase in the number of reports of AIP in recent years, the overall number of patients is still relatively small. Three series have reported the prevalence of AIP as between 5 and $6 \%$ of all patients with chronic pancreatitis [1]. Clinically, AIP is characterized by a preponderance of elderly males, jaundice as a frequent initial symptom, an association with various sclerosing extrapancreatic lesions, such as sclerosing cholangitis, sclerosing sialadenitis, and retroperitoneal fibrosis, showing similar histological findings to the pancreas with elevated serum IgG or IgG4 levels. Therefore, AIP can be considered to be a pancreatic lesion of IgG4-related systemic disease, and its extrapancreatic lesions are clinical manifestations of organs involved in this systemic disease. AIP sometimes forms a pancreatic mass lesion, which can often be difficult to distinguish from pancreatic cancer, and this diagnostic uncertainty can lead to pancreatic resection for benign disease. We report a case of AIP with the formation of a mass mimicking neuroendocrine tumor (NET) or pancreatic cancer without any specific findings and review the relevant literature.

\section{Case Report}

In December 2007, a 53-year-old man was referred to our hospital for further evaluation of a pancreatic mass detected on ultrasonography at a medical check-up. He denied abdominal pain, nausea, vomiting, or weight loss. Vital signs were stable and the findings of physical examination were unremarkable. He had no history of alcohol consumption, smoking, or family history of pancreatic or any other gastrointestinal disease. The results of the laboratory test were almost within normal limits except for slightly elevated gastrin. Levels of pancreatic hormones such as insulin and glucagon were within the normal ranges. IgG serum level was normal and antinuclear antibody test was negative (table 1). Abdominal ultrasonography showed a 15-mm hypoechoic mass located in the pancreatic

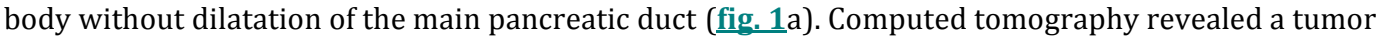
in the pancreatic body without any contrast enhancement (fig. 1b). Magnetic resonance imaging demonstrated the mass to be hyperintense on diffusion-weighted image (fig. 1c). Endoscopic retrograde cholangiopancreatography revealed slight dilatation of a branch of the pancreatic duct in the pancreatic body without stricture of the main pancreatic duct (fig. 1d). The common bile duct seemed intact, and cytology of the pancreatic juice was class II. Endoscopic ultrasonography revealed a partially encapsulated hypoechoic mass in the body of the pancreas abutting the splenic vein. On the basis of these findings, a non-functioning NET or malignant neoplasm were suspected, and laparotomy was performed. At laparotomy, an elastic firm mass well circumscribed by a thin wall suggestive of a NET was found at the pancreatic body. Therefore enucleation was performed (fig. 2a). The tumor could be easily dissected. Intraoperative frozen section revealed no evidence of malignancy, thus further excision was not attempted. Histologically, the tumor was partially indiscrete and showed extensive fibrosis, and demonstrated infiltration of lymphocytes, plasma cells, and eosinophils with lymphoid follicles around the pancreatic duct and pancreatic parenchyma, and obliterative venulitis, which are typical features of AIP (fig. 2b). Subsequently, immunohistological examination demonstrated that the pancreatic duct was surrounded by abundant IgG4-positive plasma cells (fig. 2c). On the basis of these findings, the tumor was diagnosed as AIP. Postoperatively, the level of serum IgG increased but then decreased to normal limits without any treatment, and the patient is currently doing well.

\section{Discussion}

AIP is a particular type of pancreatitis that is thought to have an autoimmune etiology. Since Yoshida et al. proposed AIP as a disease entity in 1995, many cases of AIP have been reported in Japan, and AIP has received increasing awareness and better understanding worldwide [2]. The diagnosis of focal forms can be difficult as AIP may 
mimic pancreatic adenocarcinoma [3-5]. Therefore, there have been several cases of AIP in whom resection was performed because of a high suspicion of pancreatic cancer $[6,7]$. In the past decade, many different diagnostic criteria for AIP have been proposed from Asia, Europe and North America [8-11]. In 2002, diagnostic criteria for AIP were proposed first in the world by the Japan Pancreas Society and revised in 2006 [8, 9]. These criteria are based on the minimum consensus of AIP and aim to avoid misdiagnosing pancreatic cancer as much as possible, but not to screen for AIP. These criteria consist of the following radiological, serological, and histopathological items: (1) radiological imaging showing narrowing of the main pancreatic duct and enlargement of the pancreas, which are characteristic of the disease; (2) laboratory data showing abnormally elevated levels of serum gamma globulin, IgG or IgG4, or the presence of autoantibodies; (3) histopathological examination of the pancreas demonstrating marked fibrosis and prominent infiltration of lymphocytes and plasma cells, which is called lymphoplasmacytic sclerosing pancreatitis (LPSP). For a diagnosis of AIP, criterion 1 must be present, together with criterion 2 and/or criterion 3. However, it is necessary to exclude malignant diseases such as pancreatic or biliary cancer. In 2006, two new sets of diagnostic criteria for AIP were proposed, in Korea and the United States (Mayo Clinic), respectively $[10,11]$. These criteria included 'response to steroid' as one of the diagnostic items. When response to steroid therapy is added to the criteria, the diagnostic sensitivity is increased. However, in the diagnostic criteria in Japan, 'response to steroid' is excluded. It is possible that the ready use of steroids by general physicians inexperienced in pancreatic disease will delay the diagnosis of pancreatic cancer, which may lead to cancer progression in some cases [12]. We agree that a trial of steroid therapy should be avoided in the absence of the typical features of AIP, such as in our case, for whom non-functioning NET or pancreatic cancer were suspected without considering AIP in the differential diagnosis, and laparoto my was performed because no specific findings in the criteria of AIP had been seen. Endoscopic ultrasonography-guided fine-needle aspiration is frequently used worldwide to rule out pancreatic cancer, however its yield for cancer is not perfect, and negative biopsy does not rule out cancer $[13,14]$.

In 2011, the international consensus diagnostic criteria for AIP were proposed. AIP was classified into two types [15]. In one form the histological description is called LPSP or AIP without granulocyte epithelial lesions. The other form is idiopathic duct-centric pancreatitis (IDCP) or AIP with granulocyte epithelial lesions. Clinically, LPSP seems to be the pancreatic manifestation of an IgG4-related systemic disease characterized by elevated serum IgG4 levels and extrapancreatic lesions such as sclerosing cholangitis, sclerosing sialadenitis, and retroperitoneal fibrosis associated with infiltration with abundant IgG4-positive plasma cells. In contrast, IDCP usually has no or very few IgG4-positive plasma cells, although this can vary. IDCP does not seem to be a systemic disease; rather, it seems to be a pancreas-specific disorder. Since IDCP patients are seronegative and lack other organ involvement, a definitive diagnosis requires pancreatic histology.

The present case seems to be LPSP because of histological features of abundant IgG4-positive cells infiltrating the pancreatic duct. The patient therefore is followed up as an outpatient, with special attention for the appearance of extrapancreatic lesions. Although pancreatic tumor in the present case was incidentally detected during medical check-up, he was asymptomatic, seronegative and revealing atypical features 
on imaging, which seems to be a rare presentation of AIP. Clinically, this type of AIP is difficult to diagnose or to differentiate from pancreatic cancer.

In conclusion, we report a rare case of histopathologically proven AIP in whom the lesion was small and no characteristic findings were obtained by imaging or blood tests.

\section{Disclosure Statement}

The authors have no conflict of interest.

Table 1. Laboratory data on admission

\begin{tabular}{ll}
\hline Total bilirubin & $1.6 \mathrm{mg} / \mathrm{dl}$ \\
Direct bilirubin & $0.4 \mathrm{mg} / \mathrm{dl}$ \\
Alkaline phosphatase & $287 \mathrm{IU} / \mathrm{l}$ \\
Gamma-glutamyl transpeptidase & $36 \mathrm{IU} / \mathrm{l}$ \\
Amylase & $91 \mathrm{IU} / \mathrm{l}$ \\
Lipase & $24 \mathrm{IU} / \mathrm{l}$ \\
Trypsin & $410 \mathrm{IU} / \mathrm{l}$ \\
Elastase-1 & $160 \mathrm{IU} / \mathrm{l}$ \\
CA19-9 & $11 \mathrm{ng} / \mathrm{ml}$ \\
DUPAN-2 & $<25 \mathrm{U} / \mathrm{ml}$ \\
SPan-1 & $8.1 \mathrm{U} / \mathrm{ml}$ \\
Glucagon & $92 \mathrm{pg} / \mathrm{ml}$ \\
Gastrin & $340 \mathrm{pg} / \mathrm{ml}$ \\
Insulin & $4 \mathrm{mU} / \mathrm{ml}$ \\
IgG & $1,260 \mathrm{mg} / \mathrm{dl}$ \\
Antinuclear antibody test & $n e g a t i v e$
\end{tabular}



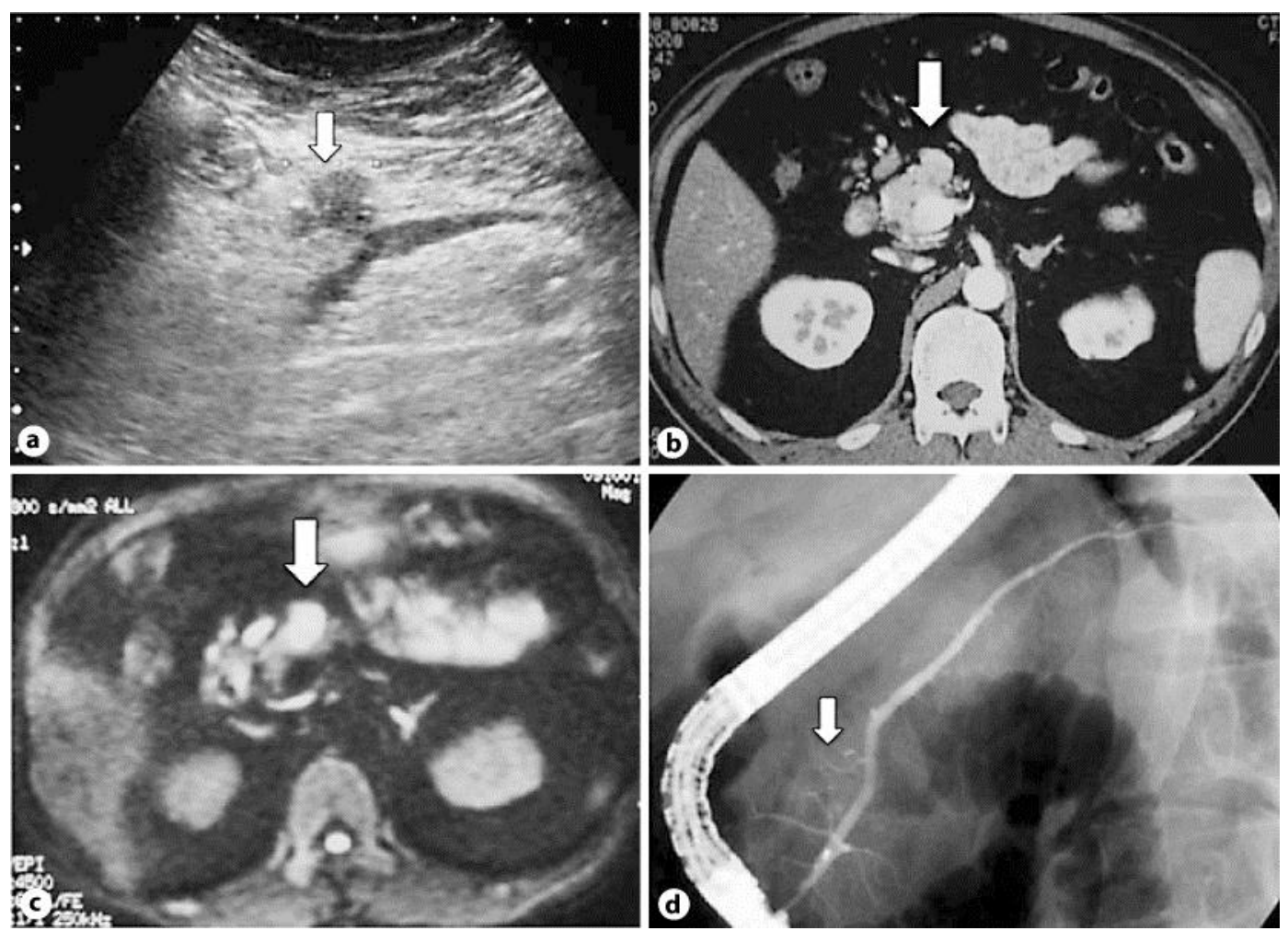

Fig. 1. a Abdominal ultrasonography showed a $15 \mathrm{~mm}$ hypoechoic mass located in the pancreatic body without dilatation of the main pancreatic duct (arrow). $\mathbf{b}$ Computed tomography revealed a tumor in the pancreatic body without any contrast enhancement (arrow). c Magnetic resonance imaging demonstrated the mass to be hyperintense on diffusion-weighted image (arrow). $\mathbf{d}$ Endoscopic retrograde cholangiopancreatography revealed slight dilatation of a branch of the pancreatic duct in the pancreatic body without stricture of the main pancreatic duct and the common bile duct (arrow). 


\begin{tabular}{r|l|l|l}
$\begin{array}{r}\text { Case Reports in } \\
\text { Gastroenterology }\end{array}$ & $\begin{array}{l}\text { Case Rep Gastroenterol 2012;6:40-46 } \\
\text { DOI: 10.1159/000336199 }\end{array}$ & $\begin{array}{l}\text { Published online: } \\
\text { January 20, 2012 }\end{array}$ & $\begin{array}{l}\text { @ 2012 S. Karger AG, Basel } \\
\text { ISSN 1662-0631 } \\
\text { www.karger.com/crg }\end{array}$ \\
\hline
\end{tabular}
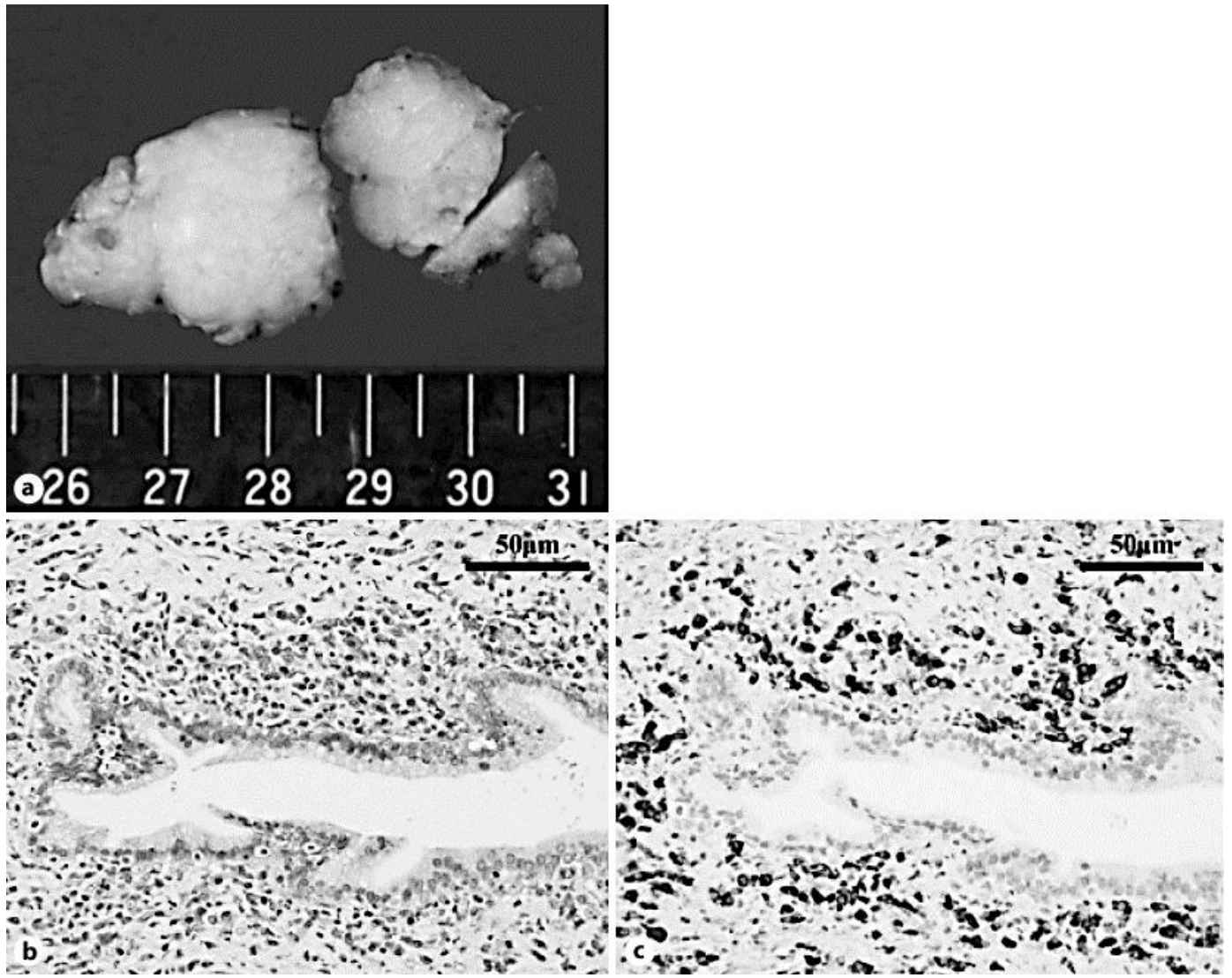

Fig. 2. a Macroscopically, a well-circumscribed solid and elastic firm tumor about $2 \mathrm{~cm}$ in diameter with gray-yellow coloration was found. b Histologically, the tumor was not well-circumscribed partially and showed extensive fibrosis and infiltration of lymphocytes, plasma cells, and eosinophils with lymphoid follicles around the pancreatic duct and pancreatic parenchyma, and obliterative venulitis was seen, which are typical features of AIP. H\&E staining, $\times 40$. c Immunohistological examination demonstrated that the pancreatic duct was surrounded by abundant IgG4-positive plasma cells. IgG4 staining, $\times 40$.

\section{References}

$>1$ Kim KP, Kim MH, Song MH, Lee SS, Seo DW, Lee SK: Autoimmune chronic pancreatitis. Am J Gastroenterol 2004;99:1605-1616.

-2 Yoshida K, Toki F, Takeuchi T, Watanabe S, Shiratori K, Hayashi N: Chronic pancreatitis caused by an autoimmune abnormality: proposal of the concept of autoimmune pancreatitis. Dig Dis Sci 1995;40: 1561-1568.

-3 Law R, Bronner M, Vogt D, Stevens T: Autoimmune pancreatitis: a mimic of pancreatic cancer. Cleve Clin J Med 2009;76:607-615.

4 Shimoda M, Kubota K, Sawada T, Katoh M, Furihata M, Iso Y, Ono Y, Fujimori T: Autoimmune pancreatitis diagnosed on the basis of immunohistology alone. A case report. JOP 2006;7:478-481.

5 Refaat R, Harth M, Proschek P, Lindemayr S, Vogl TJ: Autoimmune pancreatitis in an 11-year-old boy. Pediatr Radiol 2009;39:389-392.

6 Robinson LS, Cannon CL, Varadarajulu S, Eloubeidi MA, Vickers S, Wilcox CM: Autoimmune pancreatitis mimicking pancreatic cancer. J Hepatobiliary Pancreat Sci 2011;18:162-169. 
7 Kamisawa T, Egawa N, Nakajima H, Tsuruta K, Okamoto A, Kamata N: Clinical difficulties in the differentiation of autoimmune pancreatitis and pancreatic carcinoma. Am J Gastroenterol 2003;12: 2694-2699.

8 Members of the Criteria Committee for Autoimmune Pancreatitis of the Japan Pancreas Society: Diagnostic criteria for autoimmune pancreatitis by the Japan Pancreas Society (in Japanese). J Jpn Pancreas Soc 2002;17:585-587.

-9 Kamisawa T, Okazaki K, Kawa S: Diagnostic criteria for autoimmune pancreatitis in Japan. World J Gastroenterol 2008;14:4992-4994.

10 Kim KP, Kim MH, Kim JC, Lee SS, Seo DW, Lee SK: Diagnostic criteria for autoimmune chronic pancreatitis revisited. World J Gastroenterol 2006;12:2487-2496.

11 Chari ST, Smyrk TC, Levy MJ, Topazian MD, Takahashi N, Zhang L, Clain JE, Pearson RK, Petersen BT, Vege SS, Farnell MB: Diagnosis of autoimmune pancreatitis: the Mayo Clinic experience. Clin Gastroenterol Hepatol 2006;4:1010-1016.

12 Ohtsubo K, Watanabe H, Okada G, Tsuchiyama T, Mouri H, Yamaguchi Y, Motoo Y, Okai T, Amaya K, Kitagawa H, Ohta T, Gabata T, Matsuda K, Ohta H, Sawabu N: A case of pancreatic cancer with formation of a mass mimicking alcoholic or autoimmune pancreatitis in a young man: possibility of diagnosis by hypermethylation of pure pancreatic juice. JOP 2008;9:37-45.

13 Shakov R, DePasquale JR, Elfarra H, Spira RS: Autoimmune pancreatitis: case series and review of the literature. Ann Clin Lab Sci 2009;39:167-175.

14 Kamisawa T, Takuma K, Tabata T, Inaba Y, Egawa N, Tsuruta K, Hishima T, Sasaki T, Itoi T: Serum IgG4-negative autoimmune pancreatitis. J Gastroenterol 2011;46:108-116.

-15 Shimosegawa T, Chari ST, Frulloni L, Kamisawa T, Kawa S, Mino-Kenudson M, Kim MH, Kloppel G, Lerch MM, Lohr M, Notohara K, Okazaki K, Schneider A, Zhang L; International Association of Pancreatology: International consensus diagnostic criteria for autoimmune pancreatitis: guidelines of the International Association of Pancreatology. Pancreas 2011;40:352-358. 